\title{
Association between dietary carotenoid intakes and the risk of asthma in adults: a cross sectional study of NHANES, 2007-2012
}

Wei Zhang ( $\sim$ zhangwei2384@126.com )

Shaanxi Provincial People's Hospital https://orcid.org/0000-0001-5806-0469

\section{Yadan Wang}

Ruibiao(Wuhan) Biotechnology Co

\section{Weijie Li}

Shaanxi Provincial People's Hospital

Jie Du

Shaanxi Provincial People's Hospital

\section{Research}

Keywords: asthma, dietary carotenoid intakes, nutrition, oxidative stress

Posted Date: March 30th, 2021

DOI: https://doi.org/10.21203/rs.3.rs-338690/v1

License: (c) (i) This work is licensed under a Creative Commons Attribution 4.0 International License. Read Full License 


\section{Abstract}

Background: In recent years, with the popularization of Western diet, the prevalence of asthma has continued to rise; Oxidative stress is thought to be an important mechanism of asthma. Carotenoids, as antioxidants, may reduce the risk of asthma, but previous studies have been controversial. In this study, we aimed to investigate the association between dietary carotenoid intakes and asthma using data from a nationally representative sample of U.S. adults.

Methods: In this cross-section study, all the data were accessed from National Health and Nutrition Examination Survey (NHANES) 2007-2012. Asthma was defined by self -report questionnaires and the dietary carotenoids data were estimated from a 24 -hour recall. Weighted multivariable linear regression models and the smooth curve fittings were applied to explore the association between total carotenoid intake, dietary carotenoid subgenera including ( $\alpha$-carotene, $\beta$-carotene, $\beta$-cryptoxanthin, lutein with zeaxanthin, and lycopene) and the risk of asthma.

Results: The present study included a total of 13039 participants, 1784 of whom were defined as having current asthma. Intakes of all kinds of carotenoids were lower in those participants with current asthma. The odds ratios with $95 \%$ confidence intervals of dietary a-carotene, dietary $\beta$-carotene, dietary $\beta$ cryptoxanthin, total lutein with zeaxanthin, total lycopene and total carotenoid intake for individuals with current asthma after adjusting the confounders in Model 3 were 0.80(0.67-0.95), 0.65(0.55-0.77), $0.67(0.53-0.83), 0.70(0.56-0.87), 0.76(0.60-0.98)$ and $0.60(0.48-0.75)$ in the highest versus lowest quartile, respectively. The smooth curve fittings suggested a nonlinear relationship between total carotenoid intake and the risk of current asthma.

Conclusions: Higher intake of a-carotene, $\beta$-carotene, $\beta$-cryptoxanthin, lycopene, lutein with zeaxanthin, and total carotenoid were associated with lower odds of having current asthma in the U.S. adults.

\section{Introduction}

Asthma is a common airway inflammatory disease in adults and children affecting approximately 3.58 million people worldwide(1) Due to the changes of environment and lifestyle, the incidence of asthma has been increasing over the past few decades, with an additional 100 million asthma diagnoses expected by 2025(2). Due to frequent exacerbations, hospitalizations, and management of comorbidities, asthma increases the health care costs and results in a poor quality of life for patients. Therefore, it is necessary to identify the modifiable risk factors for the incidence and morbidity of this disease in order to decrease the disease burden of asthma.

Chronic airway inflammation and airway hyperresponsiveness are the main pathological features of asthma. More and more evidence showed that oxidative stress is involved in the pathophysiological mechanism of asthma and implicated to the inflammation and severity of asthma(3-5). Dietary epidemiological evidence suggests that Western diets, characterized by high intake of saturated fats and simple sugars, are related with an increased incidence of asthma $(6,7)$ while Mediterranean diets, 
characterized by an intake of plant-based foods (mainly vegetables and fruits) may reduce the risk of asthma through its antioxidant and anti-inflammatory effects(8).

Carotenoids, which are widely found in fruits, vegetables and seaweed species, have been shown to have anti-inflammatory and anti-oxidative stress effects and proved to be related to hypertension, nonalcoholic fatty liver disease, cancer, and other chronic diseases(9-13). A few previous studies have reported the correlation between carotenoids and asthma. The result from a cross-sectional study of 68,535 women indicated that increased intakes of carotene, tomatoes and carotenoid-rich vegetables were related to a lower risk of asthma(14). Two small-sample randomized controlled studies showed that lower carotenoids intakes were associated with higher risk of asthma and modifying the dietary intake of carotenoids can affect clinical outcomes in patients with asthma $(15,16)$. Also, dietary and serum carotenoids have also been associated with improved lung function in several other studies $(17,18)$. Conversely, from a meta-analysis of five cross-sectional studies, there was no association between higher dietary intake of $\beta$-carotene and asthma and FEV1 (Forced expiratory volume in one second)(19). However, most of the previous studies were conducted only for specific populations and the results of these studies were controversial. In addition, these studies only focused on the association between dietary carotenoids and asthma, and few evaluated the effect of supplementary carotenoids. Besides, the associations by age, gender and other individual characteristics are not well described and analyzed. Therefore, in this study, we aimed to explore the relationship between all kinds of dietary carotenoid intakes and asthma using data from the National Health and Nutrition Examination Survey (NHANES) (2007-2012), which provided a representative sample of the population in the US.

\section{Material And Methods}

\section{Data source}

The present study is a cross-section study, and all the data used for analysis were obtained from NHANES 2007-2012, which were conducted by the National Center for Health Statistics of the Centers for Disease Control and Prevention (CDC). All subjects signed the informed consent during the period of recruitment, and all the protocols were approved by the Institutional Review Board of the CDC.

\section{Patients}

In this study, we included adults older than 20 years from three independent cross-sectional waves (including 2007-2008, 2009-2010, 2011-2012). We excluded individuals who did not participate in asthma survey or did not have the reliable two 24-h dietary recall data. We also excluded participants with missing data on BMI, asthma, carotenoids intakes and total energy intake; Subjects who have extreme total energy intake (female, $<600$ or $>6000 \mathrm{kcal} /$ day; male, $<800$ or $>8000 \mathrm{kcal} /$ day) were also excluded from our study population.

\section{Asthma}


The information of asthma was collected by a self-administered questionnaire which were completed at the clinic visit. Individuals with current asthma were defined as affirmative responses to both of the following questions: "Has a doctor or other health professional ever told you that you have asthma?" and "In the past 12 months (have you/has SP) had wheezing or whistling in (your/his/her) chest?" Control subjects were defined as participants without current asthma, who answered "NO" to either question.

\section{Dietary carotenoid intakes}

In every NHANES cycle, two 24-hour dietary recall interviews which recorded the detailed dietary information about the participants were used to estimate various nutrients and total energy intake. The first diet recall interview was conducted in-person in the Mobile Examination Center (MEC), recording the type and amount of all food and beverage consumed in the 24 hours prior to the interview, and the second was conducted by telephone 3-10 days later. The intakes of energy and nutrients were calculated by the Food Survey Research Team of the United States Department of Agriculture (USAD) by using "what are we eating in the United States" (WWEIA).

In the present study, the dietary intakes of a-carotene, $\beta$-carotene, $\beta$-cryptoxanthin, lycopene and lutein with zeaxanthin were calculated by averaging over the two recall periods. In each NHANES cycle, the supplement intakes of lycopene and lutein with zeaxanthin were also collected during the same two 24hour. For this study, the intakes from supplements were also averaged over two days and the intakes of total lycopene and total lutein with zeaxanthin were calculated as the sum of dietary and supplement intakes. The total carotenoid intake was the sum of the intakes of dietary a-carotene, dietary $\beta$-carotene, dietary $\beta$-cryptoxanthin, total lycopene and total lutein with zeaxanthin. Dietary carotenoids intakes were adjusted by weight ( $\mathrm{mg} / \mathrm{kg}$ per day) and categorized into quartiles.

\section{C-reactive protein}

Serum CRP which was considered as a biomarker for systemic inflammation was measured and quantified by latex-enhanced nephelometry. The minimum limit of detection is $0.02 \mathrm{mg} / \mathrm{dL}$. Full details of the operation are available at www.cdc.gov/nchs/nhanes/. For this study, serum CRP levels were available from two of the NHANES cycles (2007-2008, 2009-2010).

\section{Other covariates}

For this analysis, we collected the following covariates: age (20-40, 40-60 and 60-80 years old), gender, ethnicity (Mexican American, other Hispanic, non-Hispanic White, non-Hispanic Black and other/Multiracial), marital status (married, living with partner, divorced, widowed, never married and separated), education level (less than high school, high school or more than high school), poverty income ratio which was used to reflect family Income, BMI, smoking status, total energy, total fat and dietary fiber.

Smoking status included never smoking, former smoking defined as "smoked at least 100 cigarettes in lifetime but does not currently smoke" and current smoking defined as "smoked at least 100 cigarettes in 
lifetime and currently smokes". Total energy, total fat and dietary fiber were also obtained from the average of two 24-hour dietary recall interviews.

\section{Statistical analysis}

In this study, we used percentages to present the categorical variables and means \pm SD or medians [interquartile range] to present the continuous variables. All the variables were compared among different groups by the weighted chi-square tests when they were categorical variables, and by the weighted linear regression models when they were continuous variables.

In each model, we used a weight suggested by the CDC to take into account the oversampling of minority and thus provide an accurate estimate of effects for the population. We used weighted multivariate linear regression models to explore the relationships between dietary carotenoid intakes and current asthma. For this study, we used three sequential models (Model 1: non adjusted model; Model 2: adjusted for age, gender, race; Model 3: adjusted for all the covariates) to control potential confounders. Then we used generalized additive model and the smooth curve fittings to evaluate the nonlinearity of dietary carotenoid intakes and current asthma after adjustment for the same covariates as the Model 3 of the multivariate linear regression models. In addition, subgroup analyses of the relationship between total dietary carotenoid intake and the risk of current asthma have been conducted to determine whether the results were stable in each subgroup.

We used the EmpowerStats software (www.empowerstats.com version R.3.4.3) and statistical software package $R$ to process and analyze all our data. A two-tailed $P$ value $\mathbb{0} .05$ was considered statistically significant.

\section{Results}

\section{Participants characteristics}

A total of 13039 participants were included in this study. The characteristics of the study population were shown in Table 1 and the flowchart of study cohort selection was shown in Figure 1. The average age of the study participants was $49.78 \pm 17.68$ years and $49.12 \%$ of participants were male. About 1784 (13.68\%) were classified as having current asthma based on a self-administered questionnaire. Subjects with current asthma had higher levels of BMI and CRP, lower levels of family Income and dietary fiber intake. The percentage of current asthma among smokers (either current or former) was higher than that among non-smokers. Intakes of all kinds of carotenoids in participants with current asthma were lower than those without current asthma.

The characteristics of participants in this study by quartile of total carotenoid intake level were shown in Supplementary Table S1. Subjects with a higher dietary total carotenoid intake had lower BMIs, lower prevalence of smoking and lower prevalence of current asthma compared to those with a lower dietary total carotenoid intake $(P<0.001)$. 
Figure 2 has shown the distribution of serum CRP levels according to quartile of total carotenoid intake in total subjects, non-current asthmatic subjects and current asthmatic subjects. The results suggested that the levels of CRP were lower for subjects with a higher dietary total carotenoid intake compared to those with a lower dietary total carotenoid intake. Among the total subjects with different grades of total carotenoid intake, CRP levels varied significantly (medium (Q1-Q3), quartile 1: $0.26(0.11-0.58)$, quartile 2: 0.20 (0.08-0.46), quartile 3: $0.18(0.07-0.41)$, quartile 4: $0.14(0.06-0.34)$, respectively, $\mathrm{P}<0.001)$. Also, among the subjects with current asthma or without current asthma, the results were similar.

\section{Association between dietary carotenoid intakes and the risk of current asthma}

The weighted odds ratios (ORs) with $95 \%$ Cls of asthma by quartiles of dietary carotenoids and total of them were shown in Table 2. In Model 1 without adjustment,

there were significant negative associations between different kinds of dietary carotenoid intakes and asthma (OR with $95 \% \mathrm{Cl}$ in the highest versus lowest quartiles: dietary a-carotene, $0.57(0.51-0.68)$; dietary $\beta$-carotene, 0.49(0.42-0.58); dietary $\beta$-cryptoxanthin, 0.58(0.49-0.70); dietary lycopene, 0.69(0.55-0.86); dietary lutein with zeaxanthin, $0.56(0.48-0.66)$; total lycopene, $0.69(0.55-0.86)$; total lutein with zeaxanthin, $0.56(0.48-0.66)$ and total carotenoid, $0.51(0.43-0.61)$, respectively). After adjustment for age, gender and race in Model 2 and after adjustment for all the confounding factors in Model 3 , the results remained stable and statistically significant. The trend test also showed significant associations between intakes of all other carotenoids with the exception of dietary a-carotene and the risk of current asthma.

We also used weighted generalized additive models and smooth curve fittings to visually assess the relationships between the intakes of all carotenoids and the risk of current asthma, which were presented in Figure 3. The results showed that the intakes of all carotenoids were negatively associated with the risk of current asthma in the adjusted models.

\section{Subgroup analyses}

The stratified analyses according to age and gender, were performed to further analyze the relationship of total carotenoid intake and the risk of current asthma in each subgroup, which were shown in Table 3 . In stratified analyses according to age, all levels of total carotenoid intake were significantly negatively associated with the risk of asthma across all age groups in model 1 (unadjusted model). In Model 3 , which adjusted for all confounders, the risk of asthma was still significantly lower in the third and fourth versus the first quartile analysis among participants aged 40-59 years. Stratified according to gender, in females, there was a significantly association between all levels of total carotenoid intake and a decreased risk of current asthma in all the models. In male, the third quartile of total carotenoid intake was associated with decreased risk of asthma in model 1 and model 2. And for the highest quartile of total carotenoid intakes, there was also a significantly association with decreased risk of asthma in all three models. 
The subgroup analyses for the relationship between total carotenoid intake and the risk of asthma stratified according to race and smoking status were shown in supplementary Table S2 and Table S3 . The results showed that in different subgroups, the relationship between the highest quartile of total carotenoid intake and the decreased risk of current asthma stably existed after careful adjustments, except for among other Hispanic and other/Multiracial participants.

\section{Discussion}

In this cross-sectional analysis, we used data from 3 waves of the NHANES (2007-2012) to investigate the relationship between dietary intakes of carotenoids (including $\alpha$-carotene, $\beta$-carotene, $\beta$-cryptoxanthin, lutein with zeaxanthin, lycopene and total carotenoid) and the risk of current asthma. To our knowledge, this is the first study to comprehensively examine the associations between different kinds of dietary carotenoid intakes and the risk of current asthma focusing on general population. We found that all carotenoid intakes were associated with lower odds of having current asthma. And after adjusting for age, sex, race and other potential confounding factors, these relationships remained significant. The results of generalized additive models and smooth curve fittings showed that there were nonlinear relationships between $\alpha$-carotene, $\beta$-carotene, $\beta$-cryptoxanthin, lutein with zeaxanthin, total carotenoid intakes and the risk of current asthma, and a linear relationship between lycopene intake and the risk of current asthma. In the stratified analysis, we found a stronger association between total carotenoid intake and the risk of asthma in the age group of 40 to 59 years old, and in non-Hispanic white, black and Mexican American adults. In addition, we found the levels of CRP were lower in subjects with a higher dietary total carotenoid intake compared to those with lower dietary total carotenoid intake.

Asthma is a chronic disease characterized by airway inflammation and airway hyperresponsiveness. Physiological damage caused by oxidative stress through reactive oxygen species (ROS) attack plays an important role in chronic inflammation of asthma(20). ROS increases the vagal tone by decreasing $\beta$ adrenaline function, resulting in airway hyperresponsiveness(21). ROS induces the contraction of airway smooth muscles by increasing acetylcholine and promotes the histamine release from mast cells and the mucus secretion from airway epithelial cells $(22,23)$. In addition, excessive ROS production may lead to an imbalance of respiratory antioxidants/oxidants, leading to the emergence and persistence of pulmonary fibrosis induced by TGF- $\beta 1$ (transforming growth factor- $\beta 1)(24)$. So, pulmonary and systemic oxidative stress increase the inflammatory response associated with asthma and allergies(25). Carotenoids as diet-derived antioxidants have antioxidant effects and can lower ROS activities, thereby reducing airway inflammation and improving lung function in asthmatic patients. The previous studies have shown that $\beta$-carotene can scavenge the highly reactive free radical superoxide anions and donate an electron to reduce free radical molecules to prevent oxidative damage $(8,26)$. In addition, lycopene has been shown to suppresses Th2 responses, reduce eosinophilic infiltrates in the lungs, and reduce ovalbumin-induced airway inflammation in an animal model of allergic asthma(27, 28). In our study, the results showed that total carotenoid intake was negatively associated with CRP levels in peripheral blood of participants. CRP has been shown to be significantly elevated in the airways and peripheral blood of 
asthmatic patients in previous study(29). Therefore, we hypothesize that carotenoids may promote asthma prevention through general anti-inflammatory and antioxidant capabilities.

Several meta-analyses have suggested that fruits and vegetables consumption may reduce the risk of asthma due to their antioxidant effects $(30,31)$. There are also some studies suggesting that the Mediterranean diet which is rich in vegetables and fruits had a negative correlation with the risk of asthma compared with the Western $\operatorname{diet}(6,7)$. As vegetables and fruits are main sources of carotenoids, these studies indirectly suggest that carotenoid intakes reduced the risk of asthma. Two previous studies on serum antioxidant levels and asthma risk using NHANES(1988-1994) among children and adults respectively showed that patients with asthma had lower serum carotenoid levels than normal subjects $(32,33)$. In children, low serum carotenoid levels increased the risk of asthma(32).

Similarly, a result from a large E3N study of 68,535 French women showed that increased consumption of tomatoes, carrots and leafy vegetables (rich in carotenoids) was associated with a lower risk of asthma in women(14). Recently, a cross-sectional observational study of 158 adolescents found that obese asthmatics had the lowest serum total carotenoid level compared to healthy weight asthmatics and healthy weight controls. They speculated that carotenoids may have a protective effect on metabolic health and lung function in obese asthmatic patients(34). Results from both a randomized controlled study(35) and a case-controlled study (36)showed that dietary supplementation or adequate intake of lycopene-rich foods may be beneficial in asthmatic subjects, improving lung function and decreasing serum CRP levels in asthmatic patients. In contrast, a meta-analysis (19) which include five observational studies suggested that high dietary $\beta$-carotene intake was not associated with the risk of asthma and lung function. However, all five studies included only $\beta$-carotene, without other types of carotene consumption and two studies lacked adjustment for the potentially important confounding factors.

Our study used a larger and nationally representative NHANES database to investigate the association between the intakes of multiple carotenoids singly and in combination and the risk of asthma. We also adjusted for more potential confounders than previous studies, including modifications of lumped dietary fiber and fatty acids, which have been reported to be associated with the risk of asthma $(37,38)$. Further stratification found that the association was stronger in the age group of 40 to 59 years old, and in nonHispanic white, black and Mexican American adults. And we first used a generalized additive model and smooth curve fitting to show the linear and nonlinear relationship of the intakes of carotenoids and the risk of current asthma. Furthermore, we analyzed the relationship between carotenoids and CRP levels, providing a possible mechanism. These are improvements on previous studies.

In the present study, there were some limitations. Frist, just like any other cross-sectional study, the results presented in this study were associations and do not provide causality, and the molecular and physiological mechanisms behind it need to be further studied and clarified. Second, the average carotenoid intakes in this study were calculated by means of two 24 -hour dietary recalls, so there may be recall bias. And we used self-reported scale instead of lung function to identify participants with asthma, which may lead to misclassification. Third, we used CRP as a marker of systemic inflammation, but the 
lack of information on active infection and medication which can alter CRP levels may affect the accuracy of the results. Fourth, although we have adjusted some confounding factors, there are still some potentially unknown confounding factors that may affect our results.

\section{Conclusion}

In conclusion, our investigation indicates that in a sample of U.S. adults, higher intakes of a-carotene, $\beta$ carotene, $\beta$-cryptoxanthin, lutein with zeaxanthin, lycopene or total of them were associated with lower risk of current asthma and lower systemic inflammation. The association between total dietary carotenoid intake and the risk of asthma was stronger among the age group of 40 to 59 years old, and in non-Hispanic white, black and Mexican American adults. The relationship needs to be further validated in other prospective studies and the mechanisms behind these associations need to be further investigated. These findings may help people reduce asthma risk and get the disease better controlled by changing their lifestyle and diet factors.

\section{Abbreviations}

NHANES: The National Health and Nutrition Examination Surveys; FEV1: Forced expiratory volume in one second; MEC: Mobile Examination Center; CRP: C-reactive protein; PIR: Poverty income ratio; BMI: Body mass index; ROS: reactive oxygen species; TGF- $\beta 1$ : transforming growth factor- $\beta 1$; OR: Odds ratio; $\mathrm{Cl}$ : Confidence interval;

\section{Declarations}

\section{Acknowledgments}

The authors thank the investigators and participants of the National Health 361

and Nutrition Examination Survey, the parent study, who made this report possible.

\section{Authors' Contributions}

Wei Zhang and Jie Du designed the research; Wei Zhang and Yadan wang analyzed the data; Wei Zhang and Wejjie Li wrote the paper. All authors contributed to data collection and edited the manuscript. All authors provided critical revisions of the manuscript and approved the final manuscript.

\section{Funding}

This research was funded by Key Research and Development Project of Shaanxi Province 353 (2020ZDLSF01-06)

\section{Availability of data and materials}


Data used in the study is publicly available through the NHANES database (available at https://wwwn.cdc.gov/nchs/nhanes/continuousnhanes/default.aspx).

\section{Ethics approval and consent to participate}

Not applicable.

\section{Consent for publication}

Not applicable.

\section{Competing interests}

The authors declare that they have no competing interests.

\section{References}

1. Global, regional, and national age-sex specific mortality for 264 causes of death, 1980-2016: a systematic analysis for the Global Burden of Disease Study 2016. Lancet, 390(10100), 1151-1210 (2017) doi:10.1016/S0140-6736(17)32152-9

2. S. C. Dharmage, J. L. Perret and A. Custovic: Epidemiology of Asthma in Children and Adults. Front Pediatr, 7, 246 (2019) doi:10.3389/fped.2019.00246

3. H. M. Ochs-Balcom, B. J. B. Grant, P. Muti, C. T. Sempos, J. L. Freudenheim, R. W. Browne, S. E. McCann, M. Trevisan, P. A. Cassano, L. lacoviello and H. J. Schünemann: Antioxidants, oxidative stress, and pulmonary function in individuals diagnosed with asthma or COPD. Eur. J. Clin. Nutr., 60(8), 991-9 (2006)

4. R. M. Andrianasolo, E. Kesse-Guyot, M. Adjibade, S. Hercberg, P. Galan and R. Varraso: Associations between dietary scores with asthma symptoms and asthma control in adults. The European respiratory journal, 52(1) (2018) doi:10.1183/13993003.02572-2017

5. M. Andrianjafimasy, F. Zerimech, Z. Akiki, H. Huyvaert, N. Le Moual, V. Siroux, R. Matran, O. Dumas and R. Nadif: Oxidative stress biomarkers and asthma characteristics in adults of the EGEA study. The European respiratory journal, 50(6) (2017) doi:10.1183/13993003.01193-2017

6. C. Frontela-Saseta, C. A. González-Bermúdez and L. García-Marcos: Diet: A Specific Part of the Western Lifestyle Pack in the Asthma Epidemic. Journal of clinical medicine, 9(7) (2020) doi: $10.3390 / \mathrm{jcm} 9072063$

7. E. P. Brigham, F. Kolahdooz, N. Hansel, P. N. Breysse, M. Davis, S. Sharma, E. C. Matsui, G. Diette and M. C. McCormack: Association between Western diet pattern and adult asthma: a focused review. Annals of allergy, asthma \& immunology : official publication of the American College of Allergy, Asthma, \& Immunology, 114(4), 273-80 (2015) doi:10.1016/j.anai.2014.11.003

8. J. Alwarith, H. Kahleova, L. Crosby, A. Brooks, L. Brandon, S. M. Levin and N. D. Barnard: The role of nutrition in asthma prevention and treatment. Nutr. Rev., 78(11), 928-938 (2020) 
doi:10.1093/nutrit/nuaa005

9. K. Christensen, C. E. Gleason and J. A. Mares: Dietary carotenoids and cognitive function among US adults, NHANES 2011-2014. Nutr. Neurosci., 23(7), 554-562 (2020) doi:10.1080/1028415X.2018.1533199

10. Z. Li, J. Chen and D. Zhang: Association between dietary carotenoid intakes and hypertension in adults: National Health and Nutrition Examination Survey 2007-2014. J. Hypertens., 37(12), 23712379 (2019) doi:10.1097/HJH.0000000000002200

11. H. Ge, T. Yang, J. Sun and D. Zhang: Associations between dietary carotenoid intakes and the risk of depressive symptoms. Food Nutr. Res., 64 (2020) doi:10.29219/fnr.v64.3920

12. K. Christensen, T. Lawler and J. Mares: Dietary Carotenoids and Non-Alcoholic Fatty Liver Disease among US Adults, NHANES 2003囚2014. Nutrients, 11(5) (2019) doi:10.3390/nu11051101

13. J. H. Kim, J. Lee, I. J. Choi, Y. Kim, O. Kwon, H. Kim and J. Kim: Dietary Carotenoids Intake and the Risk of Gastric Cancer: A Case-Control Study in Korea. Nutrients, 10(8) (2018) doi:10.3390/nu10081031

14. I. Romieu, R. Varraso, V. Avenel, B. Leynaert, F. Kauffmann and F. Clavel-Chapelon: Fruit and vegetable intakes and asthma in the E3N study. Thorax, 61(3), 209-15 (2006)

15. L. G. Wood, M. L. Garg, J. M. Smart, H. A. Scott, D. Barker and P. G. Gibson: Manipulating antioxidant intake in asthma: a randomized controlled trial. In, (2012) doi:10.3945/ajcn.111.032623

16. I. Neuman, H. Nahum and A. Ben-Amotz: Reduction of exercise-induced asthma oxidative stress by lycopene, a natural antioxidant. In, (2000)

17. L. Jun and M. Root: Association of Carotenoid Intake with Pulmonary Function. J. Am. Coll. Nutr., 1-5 (2020) doi:10.1080/07315724.2020.1815608

18. A. Guénégou, B. Leynaert, I. Pin, G. Le Moël, M. Zureik and F. Neukirch: Serum carotenoids, vitamins A and $\mathrm{E}$, and 8 year lung function decline in a general population. Thorax, 61(4), 320-6 (2006)

19. J. Gao, X. Gao, W. Li, Y. Zhu and P. J. Thompson: Observational studies on the effect of dietary antioxidants on asthma: a meta-analysis. Respirology (Carlton, Vic.), 13(4), 528-36 (2008) doi:10.1111/j.1440-1843.2008.01286.x

20. J. Checa and J. M. Aran: Airway Redox Homeostasis and Inflammation Gone Awry: From Molecular Pathogenesis to Emerging Therapeutics in Respiratory Pathology. Int. J. Mol. Sci., 21(23) (2020) doi:10.3390/ijms21239317

21. K. M. Rambacher and N. H. Moniri: The $\beta 2$-adrenergic receptor-ROS signaling axis: An overlooked component of $\beta 2$ AR function? Biochem. Pharmacol., 171, 113690 (2020) doi:10.1016/j.bcp.2019.113690

22. Y. S. Cho and H. Moon: The role of oxidative stress in the pathogenesis of asthma. Allergy, asthma \& immunology research, 2(3), 183-7 (2010) doi:10.4168/aair.2010.2.3.183

23. M. Jesenak, M. Zelieskova and E. Babusikova: Oxidative Stress and Bronchial Asthma in ChildrenCauses or Consequences? Frontiers in pediatrics, 5, 162 (2017) doi:10.3389/fped.2017.00162 
24. Y. Liu, W. Zha, Y. Ma, F. Chen, W. Zhu, A. Ge, X. Zeng and M. Huang: Galangin attenuates airway remodelling by inhibiting TGF- $\beta 1$-mediated ROS generation and MAPK/Akt phosphorylation in asthma. Sci. Rep.-UK, 5, 11758 (2015) doi:10.1038/srep11758

25. H. Moreno-Macias and I. Romieu: Effects of antioxidant supplements and nutrients on patients with asthma and allergies. The Journal of allergy and clinical immunology, 133(5), 1237-44; quiz 1245 (2014) doi:10.1016/j.jaci.2014.03.020

26. L. Guilleminault, E. J. Williams, H. A. Scott, B. S. Berthon, M. Jensen and L. G. Wood: Diet and Asthma: Is It Time to Adapt Our Message? Nutrients, 9(11) (2017) doi:10.3390/nu9111227

27. L. C. Hazlewood, L. G. Wood, P. M. Hansbro and P. S. Foster: Dietary lycopene supplementation suppresses Th2 responses and lung eosinophilia in a mouse model of allergic asthma. The Journal of nutritional biochemistry, 22(1), 95-100 (2011) doi:10.1016/j.jnutbio.2009.12.003

28. C. Lee, J. Chang, D. Moon, Y. H. Choi, I. Choi, Y. Park and G. Kim: Lycopene suppresses ovalbumininduced airway inflammation in a murine model of asthma. Biochem. Bioph. Res. Co., 374(2), 248-52 (2008) doi:10.1016/j.bbrc.2008.07.032

29. A. A. Arif, G. L. Delclos and J. Colmer-Hamood: Association between asthma, asthma symptoms and C-reactive protein in US adults: data from the National Health and Nutrition Examination Survey, 1999-2002. Respirology (Carlton, Vic.), 12(5), 675-82 (2007)

30. B. Hosseini, B. S. Berthon, P. Wark and L. G. Wood: Effects of Fruit and Vegetable Consumption on Risk of Asthma, Wheezing and Immune Responses: A Systematic Review and Meta-Analysis. Nutrients, 9(4) (2017) doi:10.3390/nu9040341

31. E. Seyedrezazadeh, M. P. Moghaddam, K. Ansarin, M. R. Vafa, S. Sharma and F. Kolahdooz: Fruit and vegetable intake and risk of wheezing and asthma: a systematic review and meta-analysis. Nutr. Rev., 72(7), 411-28 (2014) doi:10.1111/nure.12121

32. R. I. Harik-Khan, D. C. Muller and R. A. Wise: Serum vitamin levels and the risk of asthma in children. In, (2004)

33. E. S. Ford, D. M. Mannino and S. C. Redd: Serum antioxidant concentrations among U.S. adults with self-reported asthma. The Journal of asthma : official journal of the Association for the Care of Asthma, 41(2), 179-87 (2004)

34. T. A. M. Tobias, L. G. Wood and D. Rastogi: Carotenoids, fatty acids and disease burden in obese minority adolescents with asthma. In, (2019) doi:10.1111/cea.13391

35. L. G. Wood, M. L. Garg, H. Powell and P. G. Gibson: Lycopene-rich treatments modify noneosinophilic airway inflammation in asthma: proof of concept. Free Radical Res., 42(1), 94-102 (2008) doi:10.1080/10715760701767307

36. G. Riccioni, T. Bucciarelli, B. Mancini, C. Di llio, R. Della Vecchia and N. D'Orazio: Plasma lycopene and antioxidant vitamins in asthma: the PLAVA study. The Journal of asthma : official journal of the Association for the Care of Asthma, 44(6), 429-32 (2007)

37. M. A. Saeed, K. C. Gribben, M. Alam, E. R. Lyden, C. K. Hanson and T. D. LeVan: Association of Dietary Fiber on Asthma, Respiratory Symptoms, and Inflammation in the Adult National Health and 
Nutrition Examination Survey Population. Annals of the American Thoracic Society, 17(9), 1062-1068 (2020) doi:10.1513/AnnalsATS.201910-7760C

38. L. Salaye, I. Bychkova, S. Sink, A. J. Kovalic, M. S. Bharadwaj, F. Lorenzo, S. Jain, A. V. Harrison, A. T. Davis, K. Turnbull, N. T. Meegalla, S. Lee, R. Cooksey, G. L. Donati, K. Kavanagh, H. L. Bonkovsky and D. A. McClain: A Low Iron Diet Protects from Steatohepatitis in a Mouse Model. Nutrients, 11(9) (2019) doi:10.3390/nu11092172

\section{Tables}

Table 1. Baseline characteristics of participants by current asthma, National Health and Nutrition Examination Survey 2007-2012 (N=13039) 


\begin{tabular}{|c|c|c|c|}
\hline & $\begin{array}{l}\text { Without current } \\
\text { asthma }\end{array}$ & $\begin{array}{l}\text { Current } \\
\text { asthma }\end{array}$ & $P$ \\
\hline Number of participants (\%) & $11255(86.32 \%)$ & $\begin{array}{l}1784 \\
(13.68 \%)\end{array}$ & \\
\hline NHANES cycle & & & 0.5617 \\
\hline 2007-2008 & 3687 (32.76\%) & $\begin{array}{l}621 \\
(34.81 \%)\end{array}$ & \\
\hline $2009-2010$ & 4122 (36.62\%) & $\begin{array}{l}607 \\
(34.02 \%)\end{array}$ & \\
\hline 2011-2012 & 3446 (30.62\%) & $\begin{array}{l}556 \\
(31.17 \%)\end{array}$ & \\
\hline Age & & & 0.8498 \\
\hline$<40$ & $3782(33.60 \%)$ & $\begin{array}{l}523 \\
(29.32 \%)\end{array}$ & \\
\hline$\geq 40,<60$ & 3702 (32.89\%) & $\begin{array}{l}657 \\
(36.83 \%)\end{array}$ & \\
\hline$\geq 60$ & 3771 (33.51\%) & $\begin{array}{l}604 \\
(33.86 \%)\end{array}$ & \\
\hline Gender & & & 0.2244 \\
\hline Male & $5545(49.27 \%)$ & $\begin{array}{l}860 \\
(48.21 \%)\end{array}$ & \\
\hline Female & $5710(50.73 \%)$ & $\begin{array}{l}924 \\
(51.79 \%)\end{array}$ & \\
\hline Race/Ethnicity & & & 0.6802 \\
\hline Mexican American & $1779(15.81 \%)$ & $162(9.08 \%)$ & \\
\hline Other Hispanic & 1133 (10.07\%) & $156(8.74 \%)$ & \\
\hline Non-Hispanic White & $5188(46.10 \%)$ & $\begin{array}{l}947 \\
(53.08 \%)\end{array}$ & \\
\hline Non-Hispanic Black & $2278(20.24 \%)$ & $\begin{array}{l}411 \\
(23.04 \%)\end{array}$ & \\
\hline Other/Multiracial & 877 (7.79\%) & $108(6.05 \%)$ & \\
\hline Education Level & & & 0.4211 \\
\hline Less than high school & $2760(24.52 \%)$ & $\begin{array}{l}525 \\
(29.43 \%)\end{array}$ & \\
\hline High school & $2508(22.28 \%)$ & $\begin{array}{l}457 \\
(25.62 \%)\end{array}$ & \\
\hline More than high school & $5972(53.06 \%)$ & $\begin{array}{l}801 \\
(44.90 \%)\end{array}$ & \\
\hline Missing & $15(0.13 \%)$ & $1(0.06 \%)$ & \\
\hline Marital status (\%) & & & 0.0002 \\
\hline
\end{tabular}


Married

Widowed

Divorced

Separated

Never married

Living with partner

Family Income (poverty income ratio, PIR)

Body Mass Index (kg/m2)

Waist Circumference (cm)

CRP (2007-2010)

Smoking Status

Never

Current

Former

Missing

Dietary Measures

Total Energy (kcal/day)

Total fat (gm)

Dietary fiber (gm)

Dietary $\alpha$-carotene ( $\mu \mathrm{g} / \mathrm{kg}$ per day)

Dietary $\beta$-carotene ( $\mu \mathrm{g} / \mathrm{kg}$ per day)

Dietary $\beta$-cryptoxanthin ( $\mu \mathrm{g} / \mathrm{kg}$ per day)

Dietary lycopene ( $\mu \mathrm{g} / \mathrm{kg}$ per day)

Dietary lutein/zeaxanthin ( $\mu \mathrm{g} / \mathrm{kg}$ per day)

Total Lycopene (Diet and Supplement $\mu \mathrm{g} / \mathrm{kg}$ per day)

Total Lutein/Zeaxanthin (Diet and

Supplement $\mu \mathrm{g} / \mathrm{kg}$ per day)
$6067(53.92 \%) \quad 823$

(46.21\%)

$147(8.25 \%)$

264

$(14.82 \%)$

$79(4.44 \%)$

296

(16.62\%)

$172(9.66 \%)$

$2.20 \pm 1.59<0.0001$

$31.14 \pm 8.14<0.0001$

$87.64 \pm$

$<0.0001$

23.97

$0.57 \pm 1.02$

$<0.0001$

$<0.0001$

$6493(57.72 \%) \quad 613$

(34.36\%)

$2823(25.09 \%) \quad 481$

(26.96\%)

$1934(17.19 \%) \quad 690$

(38.68\%)

$5(0.04 \%)$

$0(0.00 \%)$

$2069.08 \pm$

763.19

$2130.49 \pm$

0.1053

835.74

$76.83 \pm 35.55$

$79.85 \pm$

0.0797

37.57

$17.40 \pm 8.90$

$15.91 \pm 8.41$

$<0.0001$

$5.95 \pm 15.49$

$4.51 \pm 11.26$

0.0012

$30.82 \pm 53.64$

$23.27 \pm$ $<0.0001$

37.72

$1.25 \pm 3.05$

$1.00 \pm 2.99$

0.0001

$68.74 \pm 99.53$

$57.27 \pm$

0.0002

80.23

$20.86 \pm 39.73$

$16.67 \pm$

31.07

$58.68 \pm$

0.0004

82.53

$23.27 \pm 53.26$

$18.77 \pm$

0.0002 
Mean \pm SD for continuous variables: $P$ value was calculated by weighted linear regression model.

Percent for Categorical variables: $P$ value was calculated by weighted chi-square test. Significant values are highlighted in bold.

Table 2. Weighted odds ratios and 95\% confidence intervals for current asthma according to quartiles of carotenoids intakes 


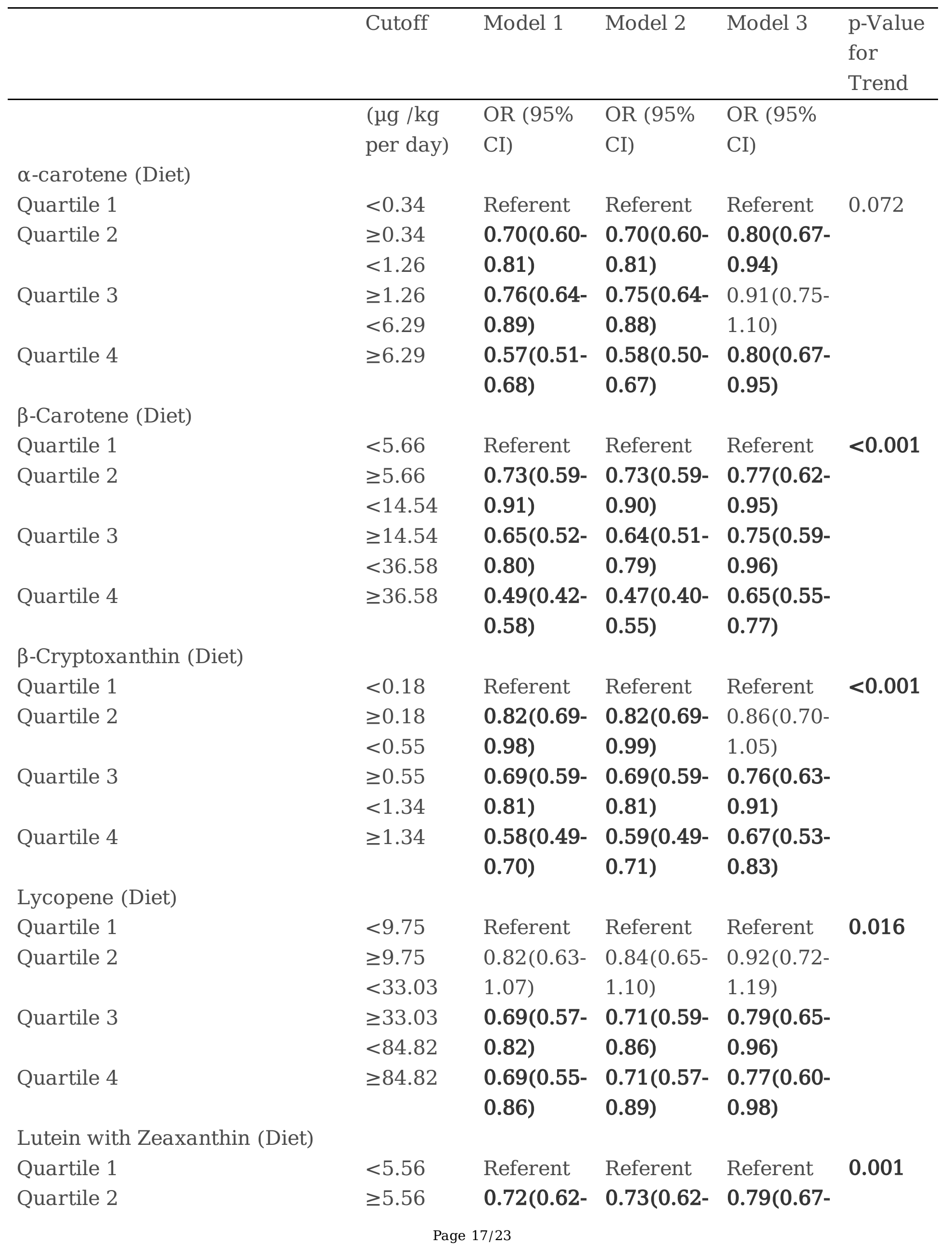




$\begin{array}{lllll} & <10.15 & 0.84) & 0.85) & 0.94 \square \\ \text { Quartile 3 } & \geq 10.15 & 0.69(0.57- & 0.69(0.57- & 0.75(0.61- \\ & <20.02 & 0.84) & 0.84) & 0.92) \\ \text { Quartile } 4 & \geq 20.03 & 0.56(0.48- & 0.54(0.46- & 0.69(0.56- \\ & & 0.66) & 0.64) & 0.85)\end{array}$

Total Lycopene (Diet and

Supplement)

Quartile 1

$<10.64$ Referent Referent Referent $\mathbf{0 . 0 1 4}$

Quartile 2

$\geq 10.64 \quad 0.84(0.65-0.86(0.66-\quad 0.94(0.73$ -

Quartile 3

$<34.09 \quad 1.10)$

1.12)

1.20)

$\geq 34.09$

$0.70(0.59-$

$0.72(0.61-$

$0.79(0.66-$

Quartile 4

$<85.93$

0.82)

$0.86)$

0.92)

$\geq 85.93$

$0.69(0.55-$

0.86)

$0.71(0.57-$

$0.76(0.60-$

Total Lutein with Zeaxanthin

(Diet and Supplement)

\begin{tabular}{llllll} 
Quartile 1 & $<5.87$ & Referent & Referent & Referent & 0.002 \\
Quartile 2 & $\geq 5.87$ & $0.77(0.64-$ & $0.77(0.64-$ & $0.82(0.68-$ & \\
& $<10.78$ & $0.91)$ & $0.92)$ & $1.00)$ & \\
Quartile 3 & $\geq 10.79$ & $0.66(0.54-$ & $0.65(0.53-$ & $0.72(0.57-$ & \\
& $<21.23$ & $0.81)$ & $0.80)$ & $0.91)$ \\
Quartile 4 & $\geq 21.24$ & $0.56(0.48-$ & $0.53(0.45-$ & $0.70(0.56-$ & \\
& & $0.66)$ & $0.64)$ & $0.87)$ & \\
Total carotene (Diet and & & & & & \\
Supplement) & & & & \\
Quartile 1 & $<41.43$ & Referent & Referent & Referent & $<0.001$ \\
Quartile 2 & $\geq 41.46$ & $0.69(0.57-$ & $0.70(0.57-$ & $0.76(0.63-$ & \\
& $<86.75$ & $0.84)$ & $0.85)$ & $0.93)$ \\
Quartile 3 & $\geq 86.75$ & $0.64(0.54-$ & $0.64(0.54-$ & $0.76(0.63-$ & \\
& $<165.58$ & $0.76)$ & $0.77)$ & $0.93)$ \\
Quartile 4 & $\geq 165.59$ & $0.51(0.43-$ & $0.51(0.42-$ & $0.60(0.48-$ & \\
& & $0.61)$ & $0.61)$ & $0.75)$ & \\
\hline
\end{tabular}

\section{Significant values are highlighted in bold.}

Model 1: Non adjusted model.

Model 2: adjusted age (continuous), gender, race (non-Hispanic white, black, Mexican American, other Hispanic, other race/ethnicity)

Model 3: age (continuous), gender, race (non-Hispanic white, black, Mexican American, other Hispanic or other race/ethnicity), education (less than high school, high school, more than high school or mission), marital status (married, widowed, divorced, separated, never 
married or living with partner), family Income (continuous), smoking status (never, current or former), intake energy (continuous), total fat (continuous) and dietary fiber (continuous).

Table 3. Weighted odds ratios (95\% confidence intervals) for current asthma according to quartiles of dietary total carotenoids intake, stratified by age and gender.

\begin{tabular}{|c|c|c|c|}
\hline Total carotenoid & Model 1 & Model 2 & Model 3 \\
\hline 20-39 years old & OR 95\% CI & OR 95\% CI & OR 95\% CI \\
\hline Quartile 1 & Referent & Referent & Referent \\
\hline Quartile 2 & $0.68(0.50-0.93)$ & $0.68(0.50-0.94)$ & $0.78(0.55-1.11)$ \\
\hline Quartile 3 & $0.66(0.48-0.91)$ & $0.67(0.49-0.92)$ & $0.78(0.54-1.12)$ \\
\hline Quartile 4 & $0.55(0.41-0.75)$ & $0.55(0.40-0.75)$ & $0.67(0.45-1.02)$ \\
\hline \multicolumn{4}{|l|}{ 40-59 years old } \\
\hline Quartile 1 & Referent & Referent & Referent \\
\hline Quartile 2 & $0.71(0.52-0.99)$ & $0.72(0.52-1.01)$ & $0.74(0.54-1.02)$ \\
\hline Quartile 3 & $0.58(0.45-0.77)$ & $0.59(0.45-0.78)$ & $0.70(0.53-0.94)$ \\
\hline $\begin{array}{l}\text { Quartile } 4 \\
\geq 60 \text { years old }\end{array}$ & $0.44(0.33-0.59)$ & $0.44(0.32-0.59)$ & $0.49(0.34-0.68)$ \\
\hline Quartile 1 & Referent & Referent & Referent \\
\hline Quartile 2 & $0.67(0.53-0.86)$ & $0.67(0.53-0.87)$ & $0.79(0.60-1.05)$ \\
\hline Quartile 3 & $0.71(0.52-0.97)$ & $0.71(0.52-0.97)$ & $0.87(0.61-1.26)$ \\
\hline Quartile 4 & $0.61(0.45-0.82)$ & $0.60(0.46-0.81)$ & $0.75(0.51-1.11)$ \\
\hline \multicolumn{4}{|l|}{ Male } \\
\hline Quartile 1 & Referent & Referent & Referent \\
\hline Quartile 2 & $0.72(0.52-1.01 \square$ & $0.73(0.52-1.01)$ & $0.80(0.56-1.15)$ \\
\hline Quartile 3 & $0.74(0.56-0.97)$ & $0.75(0.57-0.98)$ & $0.86(0.63-1.17)$ \\
\hline Quartile 4 & $0.57(0.42-0.77)$ & $0.58(0.43-0.77)$ & $0.64(0.46-0.93)$ \\
\hline \multicolumn{4}{|l|}{ Female } \\
\hline Quartile 1 & Referent & Referent & Referent \\
\hline Quartile 2 & $0.67(0.52-0.87)$ & $0.68(0.53-0.88)$ & $0.74(0.58-0.95)$ \\
\hline Quartile 3 & $0.56(0.45-0.70)$ & $0.57(0.45-0.71)$ & $0.70(0.56-0.87)$ \\
\hline Quartile 4 & $0.46(0.36-0.61)$ & $0.46(0.35-0.61)$ & $0.57(0.42-0.77)$ \\
\hline
\end{tabular}

Each subgroup analyses adjusted for all other variables except its own.

Significant values are highlighted in bold.

Model 1: Non adjusted model.

Model 2: adjusted age, gender, race

Model 3: age, gender, race, education, marital status, family Income, smoking status, intake energy, total fat and dietary fiber 


\section{Figures}

NHANES 2007-2008

10149 individuals

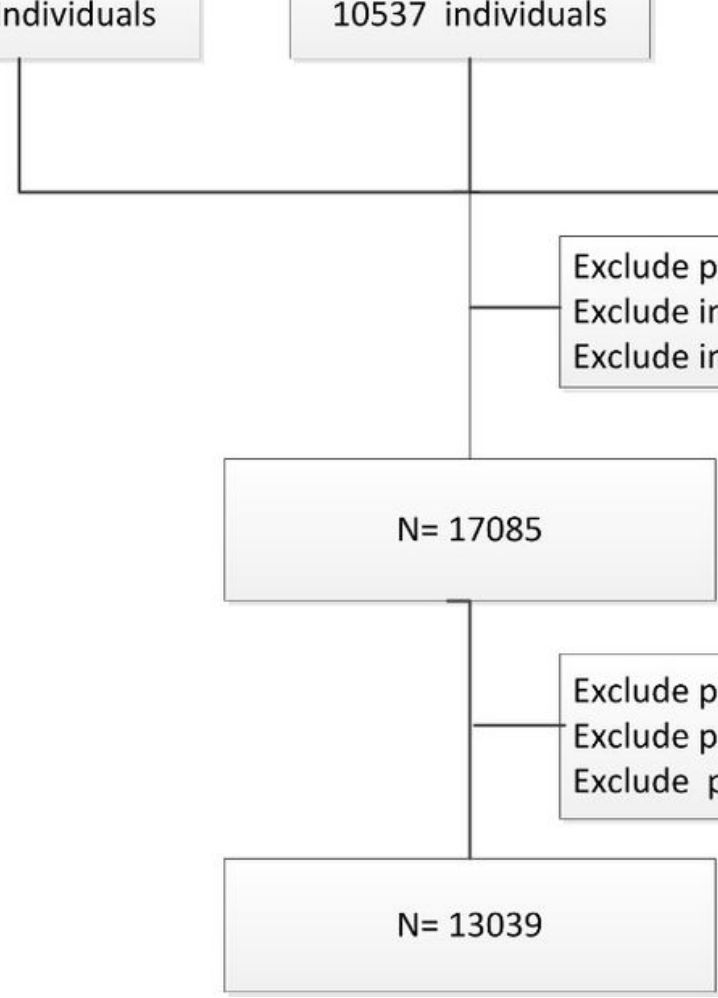

NHANES 2011-2012

9756 individuals

\section{Figure 1}

Flow chart of the current study. 


\section{Quartile $1 \square$ Quartile 2 Quartile $3 \square$ Quartile 4}

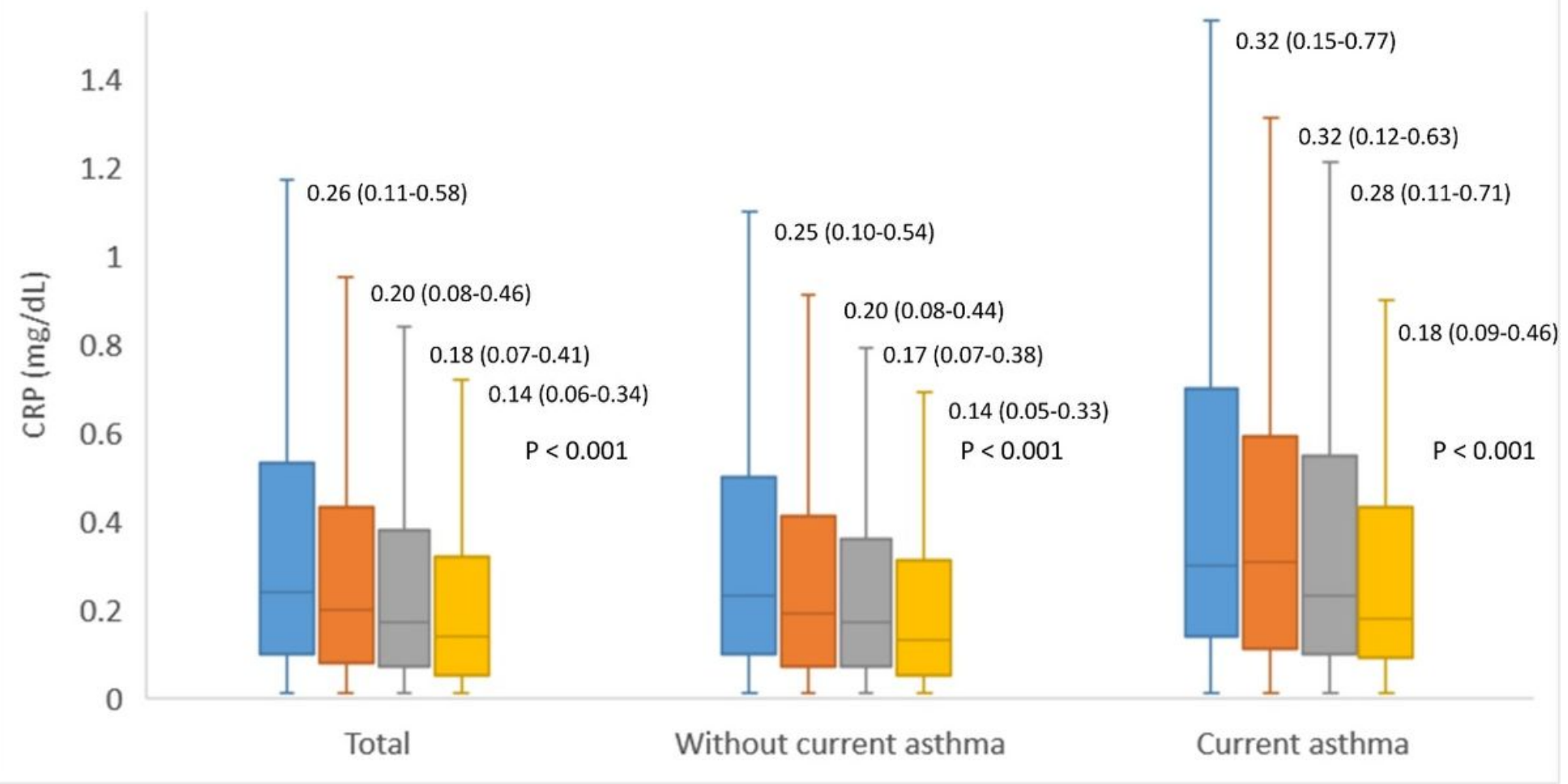

\section{Figure 2}

Levels of serum CRP according to quartile of total carotenoid intake. Boxplot shows the median (bar), the first and third quartiles (box) and the 1s and 99th percentiles (whiskers) for each group. Data are presented as medians (Q1-Q3). P value was calculated by weighted linear regression model. 

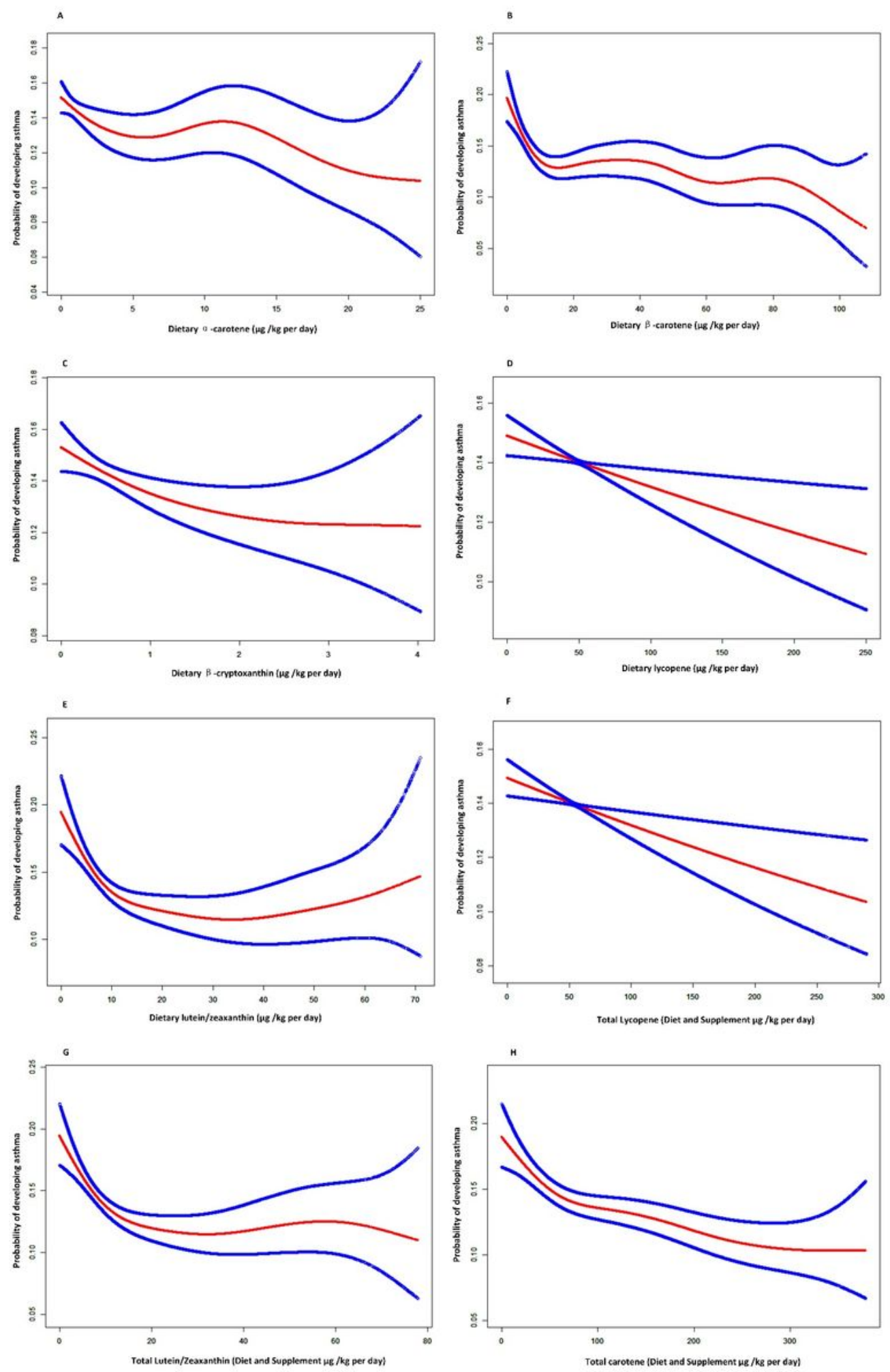

Figure 3

The adjusted smoothed plots between carotenoid intakes and current asthma based on two-piece-wise regression model. $(\mathrm{A}-\mathrm{H})$ The nonlinear or linear relationship between $\alpha$-carotene, $\beta$-carotene, $\beta$ cryptoxanthin, dietary lycopene, dietary lutein with zeaxanthin, total lycopene, total lutein with zeaxanthin and total carotenoid intakes. Adjusted: age, gender, race, education, marital status, family Income, 
smoking status, intake energy, total fat and dietary fiber. The red and blue line represent the estimated values and their corresponding $95 \%$ confidence intervals.

\section{Supplementary Files}

This is a list of supplementary files associated with this preprint. Click to download.

- Supplementtable.docx 